\title{
CONVERGENCE AND TRAVELLING FRONTS IN FUNCTIONAL DIFFERENTIAL EQUATIONS WITH NONLOCAL TERMS: A COMPETITION MODEL*
}

\author{
STEPHEN A. GOURLEY ${ }^{\dagger}$ AND SHIGUI RUAN ${ }^{\ddagger}$
}

\begin{abstract}
In this paper we consider a two-species competition model described by a reactiondiffusion system with nonlocal delays. In the case of a general domain, we study the stability of the equilibria of the system by using the energy function method. When the domain is one-dimensional and infinite, by employing linear chain techniques and geometric singular perturbation theory, we investigate the existence of travelling front solutions of the system.
\end{abstract}

Key words. competition-diffusion, equilibrium, stability, travelling front, energy function, geometric singular perturbation

AMS subject classifications. 92D25, 35K57, 35R20

DOI. $10.1137 /$ S003614100139991

1. Introduction. Let $\mathbf{R}=(-\infty, \infty)$, and let $\Omega$ be some open bounded region in $\mathbf{R}^{N}, N \leq 3$, with a smooth boundary $\partial \Omega$. Let $\partial / \partial n$ denote the outward normal derivative on $\partial \Omega$ and let $\Delta$ be the Laplacian operator. For $1 \leq p \leq \infty$, let $L^{p}(\Omega)$ denote the Banach space of measurable functions $u$ on $\Omega$ satisfying

$$
\|u\|_{p}=\left\{\begin{array}{lll}
\left(\int_{\Omega}|u(x)|^{p} d x\right)^{1 / p}<\infty & \text { if } & 1 \leq p<\infty, \\
{\operatorname{ess} \sup _{x \in \Omega}|u(x)|<\infty} & \text { if } & p=\infty .
\end{array}\right.
$$

In particular, if $p=2, L^{2}(\Omega)$ becomes a Hilbert space with the usual inner product $\langle\cdot, \cdot\rangle$ and $\|\cdot\|_{2}^{2}=\langle\cdot, \cdot\rangle$. Also, let $\|\cdot\|_{2}$ denote the norm in $L^{2}\left((0, T) ; L^{2}(\Omega ; R)\right)$, i.e.,

$$
\|u\|_{2}=\left(\int_{0}^{T}\|u(s)\|_{2}^{2} d s\right)^{1 / 2} .
$$

Let $u_{1}(t, x)$ and $u_{2}(t, x)$ denote the population densities of two competitors at time $t$ and location $x$, and let the diffusivities of the two competitors be $d_{1}$ and $d_{2}$, respectively. This paper is concerned with the following two-species Lotka-Volterra competition-diffusion model with distributed delays:

$$
\begin{aligned}
& \frac{\partial u_{1}}{\partial t}=d_{1} \Delta u_{1}+u_{1}\left(r_{1}-a_{1} u_{1}-b_{1} \int_{\Omega} \int_{-\infty}^{t} K_{1}(x, y, t-s) u_{2}(s, y) d s d y\right), \\
& \frac{\partial u_{2}}{\partial t}=d_{2} \Delta u_{2}+u_{2}\left(r_{2}-b_{2} \int_{\Omega} \int_{-\infty}^{t} K_{2}(x, y, t-s) u_{1}(s, y) d s d y-a_{2} u_{2}\right)
\end{aligned}
$$

*Received by the editors December 19, 2001; accepted for publication (in revised form) February 28, 2003; published electronically October 2, 2003.

http://www.siam.org/journals/sima/35-3/39991.html

${ }^{\dagger}$ Department of Mathematics and Statistics, University of Surrey, Guildford, Surrey GU2 7XH, UK (s.gourley@surrey.ac.uk).

${ }^{\ddagger}$ Department of Mathematics, University of Miami, P.O. Box 249085, Coral Gables, FL 331244250 (ruan@math.miami.edu). The research of this author was partially supported by the NSERC of Canada and the College of Arts and Sciences at the University of Miami. On leave from Dalhousie University, Halifax, NS, Canada. 
for $t>0, x \in \Omega$, under the homogeneous Neumann boundary conditions

$$
\frac{\partial u_{1}}{\partial n}=\frac{\partial u_{2}}{\partial n}=0, \quad x \in \partial \Omega
$$

and initial conditions

$$
u_{1}(\theta, x)=\phi_{1}(\theta, x) \geq 0, u_{2}(\theta, x)=\phi_{2}(\theta, x) \geq 0, \quad(\theta, x) \in(-\infty, 0] \times \Omega,
$$

where $\phi_{1}$ and $\phi_{2}$ are continuous functions. The parameters $r_{i}, a_{i}$, and $b_{i}, i=1,2$, are all positive constants.

The kernels $K_{i}(x, y, \sigma), i=1,2$, are nonnegative functions which are continuous in $(x, y) \in \bar{\Omega} \times \bar{\Omega}$ for each $\sigma \in[0, \infty)$ and measurable in $\sigma \in[0, \infty)$ for each pair $(x, y) \in$ $\bar{\Omega} \times \bar{\Omega}$. We assume that the kernels depend on both the spatial and the temporal variables. The delays in this type of model formulation are called spatiotemporal delays or nonlocal delays. This is a formulation that aims to account for the fact that, at previous times, individuals have not necessarily been at the same point in space. See Gourley and Britton [8] for a detailed discussion of this modelling issue on an infinite spatial domain and Gourley and So [9], who more recently have treated the finite domain case, explaining in detail why it leads to the type of delay term we are using in (1.1). See also Yamada [16] and the references cited therein. Gourley and So [9] concentrated on the one-dimensional domain $[0, \pi]$ and showed that on this domain a delayed variable $u(t, x)$, representing a population with diffusivity $d$, should be modelled in the equations by using a term of the form

$$
\int_{0}^{\pi} \int_{-\infty}^{t} G(x, y, t-s) k(t-s) u(s, y) d s d y
$$

where $k(t)$ is the weight given to the population $t$ time units ago and, in the homogeneous Neumann problem,

$$
G(x, y, t)=\frac{1}{\pi}+\frac{2}{\pi} \sum_{n=1}^{\infty} e^{-d n^{2} t} \cos n x \cos n y .
$$

In our formulation we are, for convenience, absorbing the $G$ and $k$ of each delay term into a single kernel $K_{i}(x, y, t)$. Regarding these kernels $K_{i}$, we shall assume that

$$
\int_{\Omega} K_{i}(x, y, \sigma) d x=\int_{\Omega} K_{i}(x, y, \sigma) d y=k_{i}(\sigma), \quad \sigma \geq 0,
$$

and

$$
\int_{0}^{\infty} k_{i}(\sigma) d \sigma=1, \quad \sigma k_{i}(\sigma) \in L^{1}((0, \infty) ; \mathbf{R})
$$

Assumption (1.5), that integration of $K_{i}(x, y, s)$ with respect to either $x$ or $y$ removes both the $x$ and the $y$ dependence, is easily seen to be reasonable when we have in mind that $K_{i}(x, y, t)$ is a product of the form $G(x, y, t) k(t)$, with $G$ given by (1.4) or the corresponding expression for whatever domain is under consideration.

The local existence of solutions $\left(u_{1}(t, x), u_{2}(t, x)\right)$ to (1.1)-(1.3) follows from the results in Yamada [17] or Ruan and $\mathrm{Wu}$ [11]. The comparison theorem for parabolic differential equations implies that $\left(u_{1}(t, x), u_{2}(t, x)\right)$ exists globally such that

$$
\begin{aligned}
& 0 \leq u_{1}(t, x) \leq \max \left\{\frac{r_{1}}{a_{1}}, \sup _{\theta \leq 0}\left\|\phi_{1}(\theta, \cdot)\right\|_{C(\bar{\Omega} ; R)}\right\}, \\
& 0 \leq u_{2}(t, x) \leq \max \left\{\frac{r_{2}}{a_{2}}, \sup _{\theta \leq 0}\left\|\phi_{2}(\theta, \cdot)\right\|_{C(\bar{\Omega} ; R)}\right\}
\end{aligned}
$$


for $x \in \bar{\Omega}$ and $t \in \mathbf{R}$. Also, by the strong maximum principle, if $\phi_{1}(0, x) \not \equiv 0$ and $\phi_{2}(0, x) \not \equiv 0$, then we have $u_{1}(t, x)>0, u_{2}(t, x)>0$ for all $x \in \bar{\Omega}$ and $t>0$.

Notice that system (1.1) has a trivial equilibrium $E_{0}=(0,0)$, two semitrivial spatially homogeneous equilibria

$$
E_{1}=\left(\frac{r_{1}}{a_{1}}, 0\right), \quad E_{2}=\left(0, \frac{r_{2}}{a_{2}}\right)
$$

and a positive spatially homogeneous equilibrium

$$
E^{*}=\left(\frac{r_{1} a_{2}-r_{2} b_{1}}{a_{1} a_{2}-b_{1} b_{2}}, \frac{r_{2} a_{1}-r_{1} b_{2}}{a_{1} a_{2}-b_{1} b_{2}}\right),
$$

provided that $a_{1} a_{2} \neq b_{1} b_{2}$ and either (i) $r_{2} b_{1}<r_{1} a_{2}$ and $r_{1} b_{2}<r_{2} a_{1}$ or (ii) $r_{2} b_{1}>$ $r_{1} a_{2}$ and $r_{1} b_{2}>r_{2} a_{1}$. The trivial equilibrium $E_{0}$ is of no interest here. The stability of the semitrivial equilibrium $E_{i}$ means that the $i$ th competitor $(i=1,2)$ wins the competition. These semitrivial equilibria are of considerable interest ecologically because of the possibility of a transition between the two. In fact we shall prove in this paper that, when the coexistence equilibrium $E^{*}$ is absent, a transition can occur between $E_{1}$ and $E_{2}$ in the form of a travelling wave-front solution.

Various special cases of system (1.1) have been studied by many researchers. When the delay kernels are independent of the spatial variable (i.e., when the delays are local), Ruan and $\mathrm{Wu}$ [11] studied the stability of the equilibria. See also Ruan and Zhao [12] for competition models with finite delays and Schiaffino and Tesei [13] for a nonlinear competition system. When there are no delays, the stability of the competition-diffusion model was investigated by Zhou and Pao [18]. Delayed competition models without diffusion have been studied by Cushing [2] and by Gopalsamy [6], and the monograph by $\mathrm{Wu}[15]$ provides a very comprehensive description of current research into delay-diffusion equations. When the domain $\Omega=(-\infty, \infty)$ and there are no delays, Conley and Gardner [1], Gardner [4], Kan-on [10], and Tang and Fife [14] have shown that the competition-diffusion model has travelling front solutions connecting the boundary equilibria

$$
\left(\frac{r_{1}}{a_{1}}, 0\right) \quad \text { and } \quad\left(0, \frac{r_{2}}{a_{2}}\right) .
$$

The existence of such solutions even for the nondelay problem is a highly nontrivial matter because one is seeking a heteroclinic connection between equilibria in a four-dimensional phase space. The introduction of delays increases the dimension to eight (for the particular delays we consider). However, when the delays are small, considerable progress can be achieved by the use of geometric singular perturbation theory.

In this paper we shall first discuss the stability of the equilibria $E_{1}, E_{2}$, and $E^{*}$ by using the energy function method (see Yamada $[16,17]$ ). Then, for the case when $\Omega=(-\infty, \infty)$, we will study the existence of travelling front solutions of system (1.1) connecting the two boundary equilibria $E_{1}$ and $E_{2}$.

2. Convergence. The main result of this section is a theorem on the global stability of each of the equilibria. First, we shall derive an inequality that will be needed in the proof of the main theorem. The hypotheses of this lemma are not restricted to this application (see, in particular, Gourley and So [9]). 
Lemma 2.1. Let $K(x, y, t)=G(x, y, t) k(t), x, y \in \Omega \subset \mathbf{R}^{N}$, where $k(t) \geq 0$ and $G(x, y, t)$ is the solution of

$$
\frac{\partial G}{\partial t}=d \nabla^{2} G, \quad \frac{\partial G}{\partial n}=0 \quad \text { on } \partial \Omega, \quad G(x, y, 0)=\delta(x-y) .
$$

Then

$$
\left\|\int_{\Omega} \int_{-\infty}^{t} K(x, y, t-s) u(s, y) d s d y\right\|_{2} \leq \int_{-\infty}^{t} k(t-s)\|u(s)\|_{2} d s
$$

for any function $u(t, x)$ such that $\partial u / \partial n=0$ on $\partial \Omega$.

Remark 2.2. Before we prove this lemma let us stress that $x$ and $y$ are both vectors in $\mathbf{R}^{N}$ here. For the purposes of computing $G, \nabla^{2}$ is calculated with respect to either of these vectors (say $x$ for definiteness) with the other one, $y$, held fixed. In the case considered in detail in [9], $\Omega$ is one-dimensional, $\nabla^{2}=\partial^{2} / \partial x^{2}$, and $G(x, y, t)$ is given by (1.4).

Proof of Lemma 2.1. We have

$$
\begin{aligned}
& \left\|\int_{\Omega} \int_{-\infty}^{t} K(x, y, t-s) u(s, y) d s d y\right\|_{2} \\
& =\left\|\int_{-\infty}^{t} \int_{\Omega} K(x, y, t-s) u(s, y) d y d s\right\|_{2} \\
& \leq \int_{-\infty}^{t}\left\|\int_{\Omega} K(x, y, t-s) u(s, y) d y\right\|_{2} d s \\
& =\int_{-\infty}^{t} k(t-s)\left\|\int_{\Omega} G(x, y, t-s) u(s, y) d y\right\|_{2} d s .
\end{aligned}
$$

Therefore, we want to show that

$$
\left\|\int_{\Omega} G(x, y, t-s) u(s, y) d y\right\|_{2} \leq\|u(s)\|_{2}
$$

for $s \leq t$. Let $\lambda_{k}, k=0,1,2, \ldots$, be the eigenvalues of $-d \nabla^{2}$ under homogeneous Neumann boundary conditions, with corresponding normalized (in $L^{2}$ ) eigenfunctions $\phi_{k}(x)$ so that

$$
-d \nabla^{2} \phi_{k}=\lambda_{k} \phi_{k}, \quad \frac{\partial \phi_{k}}{\partial n}=0 \quad \text { on } \partial \Omega
$$

Then $\lambda_{0}=0$ with $\phi_{0}=$ constant, and $\lambda_{k}>0$ for all other $k$. The solution $G(x, y, t)$ of (2.1) will be given by a Fourier series expansion in terms of these functions $\phi(x)$ with coefficients depending on $y$. In fact,

$$
G(x, y, t)=\sum_{n=0}^{\infty} e^{-\lambda_{n} t} \phi_{n}(x) \phi_{n}(y) .
$$

Also, $u(t, x)$ satisfies the boundary conditions and therefore can be expanded in terms of the $\phi_{n}$ :

$$
u(t, x)=\sum_{n=0}^{\infty} a_{n}(t) \phi_{n}(x)
$$


Therefore, since the $\phi_{k}$ are orthonormal,

$$
\int_{\Omega} G(x, y, t-s) u(s, y) d y=\sum_{n=0}^{\infty} a_{n}(s) e^{-\lambda_{n}(t-s)} \phi_{n}(x),
$$

and hence, by Parseval's identity,

$$
\begin{aligned}
\left\|\int_{\Omega} G(x, y, t-s) u(s, y) d y\right\|_{2} & =\left(\sum_{n=0}^{\infty} a_{n}^{2}(s) e^{-2 \lambda_{n}(t-s)}\right)^{1 / 2} \\
& \leq\left(\sum_{n=0}^{\infty} a_{n}^{2}(s)\right)^{1 / 2}=\|u(s)\|_{2}
\end{aligned}
$$

as desired. The proof is complete.

Next, we state our main theorem of this section.

THEOREM 2.3. Let $\left(u_{1}(t, x), u_{2}(t, x)\right)$ satisfy (1.1) with boundary conditions (1.2) and initial conditions $(1.3)$, with $\phi_{1}(0, x) \not \equiv 0$ and $\phi_{2}(0, x) \not \equiv 0$.

(i) If $r_{1} / r_{2}>a_{1} / b_{2}>b_{1} / a_{2}$, then $\lim _{t \rightarrow \infty}\left(u_{1}(t, x), u_{2}(t, x)\right)=\left(r_{1} / a_{1}, 0\right)$ uniformly for $x \in \bar{\Omega}$.

(ii) If $r_{1} / r_{2}<b_{1} / a_{2}<a_{1} / b_{2}$, then $\lim _{t \rightarrow \infty}\left(u_{1}(t, x), u_{2}(t, x)\right)=\left(0, r_{2} / a_{2}\right)$ uniformly for $x \in \bar{\Omega}$.

(iii) If $b_{1} / a_{2}<r_{1} / r_{2}<a_{1} / b_{2}$, then $\lim _{t \rightarrow \infty}\left(u_{1}(t, x), u_{2}(t, x)\right)=\left(u_{1}^{*}, u_{2}^{*}\right)$ uniformly for $x \in \bar{\Omega}$, where $u_{1}^{*}$ and $u_{2}^{*}$ are the components of the equilibrium $E^{*}$ given by $(1.8)$.

Proof. We prove only (i); the proofs of (ii) and (iii) are similar. To study the stability of the semitrivial equilibrium $E_{1}=\left(r_{1} / a_{1}, 0\right)$, define

$$
E\left(u_{1}\right)=\int_{\Omega}\left[u_{1}-\frac{r_{1}}{a_{1}}-\frac{r_{1}}{a_{1}} \log \frac{u_{1}}{r_{1} / a_{1}}\right] d x, \quad F\left(u_{2}\right)=\int_{\Omega} u_{2} d x .
$$

Then $E\left(u_{1}\right) \geq 0$ and $F\left(u_{2}\right) \geq 0$. For some constant $\alpha>0$ to be found later, we have

$$
\begin{aligned}
\frac{d}{d t}\left[\alpha E\left(u_{1}\right)+F\left(u_{2}\right)\right] \\
=\alpha \int_{\Omega} \frac{\partial u_{1}}{\partial t}\left(1-\frac{r_{1} / a_{1}}{u_{1}}\right) d x+\int_{\Omega} \frac{\partial u_{2}}{\partial t} d x \\
=-\alpha d_{1} \frac{r_{1}}{a_{1}} \int_{\Omega} \frac{\left|\nabla u_{1}\right|^{2}}{u_{1}^{2}} d x-\alpha a_{1} \int_{\Omega}\left(u_{1}-\frac{r_{1}}{a_{1}}\right)^{2} d x \\
\quad-\alpha b_{1} \int_{\Omega}\left(\int_{\Omega} \int_{-\infty}^{t} K_{1}(x, y, t-s) u_{2}(s, y) d s d y\right)\left(u_{1}-\frac{r_{1}}{a_{1}}\right) d x-a_{2} \int_{\Omega} u_{2}^{2}(t, x) d x \\
\quad+r_{2} \int_{\Omega} u_{2}(t, x) d x-b_{2} \int_{\Omega}\left(\int_{\Omega} \int_{-\infty}^{t} K_{2}(x, y, t-s) u_{1}(s, y) d s d y\right) u_{2}(t, x) d x \\
=-\alpha d_{1} \frac{r_{1}}{a_{1}} \int_{\Omega} \frac{\left|\nabla u_{1}\right|^{2}}{u_{1}^{2}} d x-\alpha a_{1} \int_{\Omega}\left(u_{1}-\frac{r_{1}}{a_{1}}\right)^{2} d x \\
\quad-\alpha b_{1} \int_{\Omega}\left(\int_{\Omega} \int_{-\infty}^{t} K_{1}(x, y, t-s) u_{2}(s, y) d s d y\right)\left(u_{1}-\frac{r_{1}}{a_{1}}\right) d x-a_{2} \int_{\Omega} u_{2}^{2}(t, x) d x \\
\quad-b_{2} \int_{\Omega}\left(\int_{\Omega} \int_{-\infty}^{t} K_{2}(x, y, t-s)\left(u_{1}(s, y)-\frac{r_{2}}{b_{2}}\right) d s d y\right) u_{2}(t, x) d x
\end{aligned}
$$


where we have used (1.5) and (1.6). By hypothesis, $r_{2} / b_{2}<r_{1} / a_{1}$, so

$$
\begin{aligned}
\frac{d}{d t}[ & \left.\alpha E\left(u_{1}\right)+F\left(u_{2}\right)\right] \\
\leq & -\alpha d_{1} \frac{r_{1}}{a_{1}} \int_{\Omega} \frac{\left|\nabla u_{1}\right|^{2}}{u_{1}^{2}} d x-\alpha a_{1} \int_{\Omega}\left(u_{1}-\frac{r_{1}}{a_{1}}\right)^{2} d x \\
& -\alpha b_{1} \int_{\Omega}\left(\int_{\Omega} \int_{-\infty}^{t} K_{1}(x, y, t-s) u_{2}(s, y) d s d y\right)\left(u_{1}-\frac{r_{1}}{a_{1}}\right) d x-a_{2} \int_{\Omega} u_{2}^{2}(t, x) d x \\
& -b_{2} \int_{\Omega}\left(\int_{\Omega} \int_{-\infty}^{t} K_{2}(x, y, t-s)\left(u_{1}(s, y)-\frac{r_{1}}{a_{1}}\right) d s d y\right) u_{2}(t, x) d x .
\end{aligned}
$$

Let $\langle\cdot, \cdot\rangle$ denote the standard inner product on $L^{2}(\Omega ; \mathbf{R}),\|\cdot\|_{2}^{2}=\langle\cdot, \cdot\rangle$. Then we have the following inequality:

$$
\begin{aligned}
\frac{d}{d t}\left[\alpha E\left(u_{1}\right)+F\left(u_{2}\right)\right]+\alpha d_{1} \frac{r_{1}}{a_{1}} \int_{\Omega} \frac{\left|\nabla u_{1}\right|^{2}}{u_{1}^{2}} d x+\alpha a_{1}\left\|u_{1}-\frac{r_{1}}{a_{1}}\right\|_{2}^{2}+a_{2}\left\|u_{2}\right\|_{2}^{2} \\
\leq-\alpha b_{1}\left\langle\int_{\Omega} \int_{-\infty}^{t} K_{1}(x, y, t-s) u_{2}(s, y) d s d y, u_{1}(t)-\frac{r_{1}}{a_{1}}\right\rangle \\
\quad-b_{2}\left\langle\int_{\Omega} \int_{-\infty}^{t} K_{2}(x, y, t-s)\left(u_{1}(s, y)-\frac{r_{1}}{a_{1}}\right) d s d y, u_{2}(t)\right\rangle .
\end{aligned}
$$

By Lemma 2.1, we have

$$
\begin{gathered}
\left\|\int_{\Omega} \int_{-\infty}^{t} K_{1}(x, y, t-s) u_{2}(s, y) d s d y\right\|_{2} \leq \int_{-\infty}^{t} k_{1}(t-s)\left\|u_{2}(s)\right\|_{2} d s \\
\leq \sup _{s \leq 0}\left\|u_{2}(s)\right\|_{2} \int_{t}^{\infty} k_{1}(s) d s+\int_{0}^{t} k_{1}(t-s)\left\|u_{2}(s)\right\|_{2} d s
\end{gathered}
$$

and

$$
\begin{gathered}
\left\|\int_{\Omega} \int_{-\infty}^{t} K_{2}(x, y, t-s)\left(u_{1}(s, y)-\frac{r_{1}}{a_{1}}\right) d s d y\right\|_{2} \leq \int_{-\infty}^{t} k_{2}(t-s)\left\|u_{1}(s)-\frac{r_{1}}{a_{1}}\right\|_{2} d s \\
\leq \sup _{s \leq 0}\left\|u_{1}(s)-\frac{r_{1}}{a_{1}}\right\|_{2} \int_{t}^{\infty} k_{2}(s) d s+\int_{0}^{t} k_{2}(t-s)\left\|u_{1}(s)-\frac{r_{1}}{a_{1}}\right\|_{2} d s .
\end{gathered}
$$

Thus, for any $T>0$,

$$
\begin{aligned}
& \left|\int_{0}^{T}\left\langle\int_{\Omega} \int_{-\infty}^{t} K_{1}(x, y, t-s) u_{2}(s, y) d s d y, u_{1}(t)-\frac{r_{1}}{a_{1}}\right\rangle d t\right| \\
& \leq \int_{0}^{T}\left\|u_{1}(t)-\frac{r_{1}}{a_{1}}\right\|\left\|_{2}\right\| \int_{\Omega} \int_{-\infty}^{t} K_{1}(x, y, t-s) u_{2}(s, y) d s d y \|_{2} d t \\
& \leq \sup _{s \leq 0}\left\|u_{2}(s)\right\|_{2} \sup _{0 \leq t \leq T}\left\|u_{1}(t)-\frac{r_{1}}{a_{1}}\right\|_{2} \int_{0}^{\infty} s k_{1}(s) d s \\
& \quad+\int_{0}^{T}\left\|u_{1}(t)-\frac{r_{1}}{a_{1}}\right\|_{2} \int_{0}^{t} k_{1}(t-s)\left\|u_{2}(s)\right\|_{2} d s d t .
\end{aligned}
$$


We now estimate the second term in the above as follows:

$$
\begin{aligned}
& \int_{0}^{T}\left\|u_{1}(t)-\frac{r_{1}}{a_{1}}\right\| \int_{2}^{t} k_{1}(t-s)\left\|u_{2}(s)\right\|_{2} d s d t \\
& \leq\left\|u_{1}-\frac{r_{1}}{a_{1}}\right\|_{2}\left(\int_{0}^{T}\left(\int_{0}^{t} k_{1}(t-s)\left\|u_{2}(s)\right\|_{2} d s\right)^{2} d t\right)^{1 / 2} \\
& \leq\left\|u_{1}-\frac{r_{1}}{a_{1}}\right\| \|_{2}\left(\int_{0}^{T}(\underbrace{\int_{0}^{t} k_{1}(t-s) d s}_{\leq 1})\left(\int_{0}^{t} k_{1}(t-s)\left\|u_{2}(s)\right\|_{2}^{2} d s\right) d t\right)^{1 / 2} \\
& \left.\leq\left\|u_{1}-\frac{r_{1}}{a_{1}}\right\|_{2}\left(\int_{0}^{T} \int_{0}^{t} k_{1}(t-s)\left\|u_{2}(s)\right\|_{2}^{2} d s d t\right)^{1 / 2}\right)^{1 / 2} \\
& =\left\|u_{1}-\frac{r_{1}}{a_{1}}\right\|_{2}(\int_{0}^{T}\left\|u_{2}(s)\right\|_{2}^{2} \underbrace{\int_{s}^{T} k_{1}(t-s) d t}_{\leq 1} d s)^{r_{1}} \|_{\left\|u_{2}\right\|_{2},}
\end{aligned}
$$

where $\|\cdot\|_{2}$ denotes the norm in $L^{2}\left((0, T) ; L^{2}(\Omega ; R)\right)$, i.e.,

$$
\|u\|_{2}=\left(\int_{0}^{T}\|u(s)\|_{2}^{2} d s\right)^{1 / 2} .
$$

Therefore, for any $T>0$,

$$
\begin{aligned}
& \left|\int_{0}^{T}\left\langle\int_{\Omega} \int_{-\infty}^{t} K_{1}(x, y, t-s) u_{2}(s, y) d s d y, u_{1}(t)-\frac{r_{1}}{a_{1}}\right\rangle d t\right| \\
& \quad \leq \sup _{s \leq 0}\left\|u_{2}(s)\right\|_{2} \sup _{0 \leq t \leq T}\left\|u_{1}(t)-\frac{r_{1}}{a_{1}}\right\|_{2} \int_{0}^{\infty} s k_{1}(s) d s+\left\|u_{1}-\frac{r_{1}}{a_{1}}\right\|\left\|_{2}\right\| u_{2} \|_{2} .
\end{aligned}
$$

In a similar way, we have

$$
\begin{aligned}
& \left|\int_{0}^{T}\left\langle\int_{\Omega} \int_{-\infty}^{t} K_{2}(x, y, t-s)\left(u_{1}(s, y)-\frac{r_{1}}{a_{1}}\right) d s d y, u_{2}(t)\right\rangle d t\right| \\
& \quad \leq \sup _{s \leq 0}\left\|u_{1}(s)-\frac{r_{1}}{a_{1}}\right\|_{2} \sup _{0 \leq t \leq T}\left\|u_{2}(t)\right\|_{2} \int_{0}^{\infty} s k_{2}(s) d s+\left\|u_{1}-\frac{r_{1}}{a_{1}}\right\|\left\|_{2}\right\| u_{2} \|_{2} .
\end{aligned}
$$

Integrating (2.4) over $[0, T]$ and noting that $\sup _{0<t<T}\left\|u_{2}(t)\right\|_{2}$ and $\sup _{0 \leq t \leq T} \| u_{1}(t)-$ $\frac{r_{1}}{a_{1}} \|_{2}$ can be bounded independently of $T$ (by $\left.(\overline{1.7})\right)$, we obtain that there exists a positive constant $C$ independent of $T$ such that

$$
\frac{\alpha d_{1} r_{1}}{a_{1}}\left\|\frac{\nabla u_{1}}{u_{1}}\right\|_{2}^{2}+\alpha a_{1}\left\|u_{1}-\frac{r_{1}}{a_{1}}\right\|_{2}^{2}+a_{2}\left\|u_{2}\right\|_{2}^{2} \leq C+\left(\alpha b_{1}+b_{2}\right)\left\|u_{1}-\frac{r_{1}}{a_{1}}\right\|_{2}\left\|u_{2}\right\|_{2}
$$


or, by using Young's inequality,

$$
\begin{aligned}
& \frac{\alpha d_{1} r_{1}}{a_{1}}\left\|\frac{\nabla u_{1}}{u_{1}}\right\|_{2}^{2}+\alpha a_{1}\left\|u_{1}-\frac{r_{1}}{a_{1}}\right\|_{2}^{2}+a_{2}\left\|u_{2}\right\|_{2}^{2} \\
& \quad \leq C+\left(\alpha b_{1}+b_{2}\right)\left(\frac{1}{2} \lambda\left\|u_{1}-\frac{r_{1}}{a_{1}}\right\|_{2}^{2}+\frac{1}{2 \lambda}\left\|u_{2}\right\|_{2}^{2}\right)
\end{aligned}
$$

for any $\lambda>0$. If we choose

$$
\lambda=\frac{\alpha b_{1}+b_{2}}{2 a_{2}}
$$

then $(2.9)$ reads as

$$
\frac{\alpha d_{1} r_{1}}{a_{1}}\left\|\frac{\nabla u_{1}}{u_{1}}\right\|_{2}^{2}+\alpha a_{1}\left\|u_{1}-\frac{r_{1}}{a_{1}}\right\|_{2}^{2} \leq C+\frac{\left(\alpha b_{1}+b_{2}\right)^{2}}{4 a_{2}}\left\|u_{1}-\frac{r_{1}}{a_{1}}\right\|_{2}^{2} .
$$

From (2.10) we can conclude that

$$
\left\|\frac{\nabla u_{1}}{u_{1}}\right\|_{2} \leq C_{1}
$$

and

$$
\left\|u_{1}-\frac{r_{1}}{a_{1}}\right\|_{2} \leq C_{2}
$$

for some constants $C_{1}, C_{2}$ independent of $T$, provided that $\alpha>0$ can be chosen such that

$$
2 \sqrt{\alpha a_{1} a_{2}}>\alpha b_{1}+b_{2}
$$

which is possible by the assumption $a_{1} a_{2}>b_{1} b_{2}$.

Because of (1.7) we may deduce from (2.11) that, for some constant $C_{3}$ independent of $T$,

$$
\left\|\nabla u_{1}\right\|_{2} \leq C_{3} .
$$

Since all this is for any $T>0,(2.13)$ and (2.12) imply that $u_{1}-r_{1} / a_{1} \in L^{2}\left((0, \infty) ; W^{1,2}(\Omega ; R)\right)$ and thus

$$
\lim _{t \rightarrow \infty}\left\|u_{1}(t)-\frac{r_{1}}{a_{1}}\right\|_{W^{1,2}}=0
$$

Therefore,

$$
\lim _{t \rightarrow \infty}\left\|u_{1}(t)-\frac{r_{1}}{a_{1}}\right\|_{C(\bar{\Omega} ; R)}=0
$$

We deduce $\lim _{t \rightarrow \infty}\left\|u_{2}(t)\right\|_{C(\bar{\Omega} ; R)}=0$ in a similar way (for example, $\lambda$ in (2.9) would be chosen differently). This completes the proof.

Remark 2.4. Theorem 2.3 indicates that if $r_{1} / r_{2}>a_{1} / b_{2}>b_{1} / a_{2}$, then the competitor with density $u_{1}$ wins the competition; if $r_{1} / r_{2}<b_{1} / a_{2}<a_{1} / b_{2}$, then the 
competitor with density $u_{2}$ overcompetes the one with density $u_{1}$; and if $b_{1} / a_{2}<$ $r_{1} / r_{2}<a_{1} / b_{2}$, then the two competing species coexist in the sense of existence and stability of a positive steady state. Theorem 2.3 extends Propositions 7.5-7.7 in Ruan and $\mathrm{Wu}[11]$ on competition-diffusion systems with infinite time delays, Theorem 3.1 in Zhou and Pao [18] on competition-diffusion systems, and the results in Gopalsamy [6] on competition systems with finite delays.

Remark 2.5. It is known (see Yamada [16]) that in the case of the single-species delay equation

$$
\frac{\partial u}{\partial t}=\Delta u+u\left(a-b u-\int_{-\infty}^{t} f(t-s) u(x, s) d s\right)
$$

on homogeneous Neumann boundary conditions, where $a$ and $b$ are nonnegative constants, bifurcations can occur from the nonzero homogeneous equilibrium state for certain kernels and for suitable values of the parameters $a$ and $b$, which include the requirement that $b$ be sufficiently small. However, in the competition model (1.1), bifurcations to spatially patterned or to spatiotemporal structures are not expected to occur from the equilibrium $E^{*}$, given by (1.8). Let us explain why this is so. A standard linearized analysis about the boundary equilibrium $\left(r_{1} / a_{1}, 0\right)$ shows that, regardless of the delay kernels, this equilibrium is unstable to perturbations in which $u_{2}>0$ if $r_{1} / r_{2}<a_{1} / b_{2}$. Similarly, the equilibrium $\left(0, r_{2} / a_{2}\right)$ is unstable to perturbations in which $u_{1}>0$ if $r_{1} / r_{2}>b_{1} / a_{2}$. Now, if the interior equilibrium $E^{*}$ were to lose stability and bifurcate to a spatial or spatiotemporal structure, we would expect that both boundary equilibria would remain unstable throughout this process so that they act as repellers. Yet the conditions for both boundary equilibria to be linearly unstable can be summarized as

$$
\frac{b_{1}}{a_{2}}<\frac{r_{1}}{r_{2}}<\frac{a_{1}}{b_{2}}
$$

which is precisely the condition for global convergence to $E^{*}$ given in (iii) of Theorem 2.3. Hence, bifurcations from $E^{*}$ cannot occur if the boundary equilibria are to remain unstable.

Remark 2.6. If we assume that, in the absence of the other competitor, each competitor's growth is governed by a Volterra integrodifferential equation with both instantaneous and delay self-regulatory terms (see Cushing [2], Schiaffino and Tesei [13], and Yamada [17]), then we have a more general model of the following form:

$$
\begin{aligned}
\frac{\partial u_{1}}{\partial t}= & d_{1} \Delta u_{1}+u_{1}\left(r_{1}-a_{1} u_{1}-c_{1} \int_{\Omega} \int_{-\infty}^{t} H_{1}(x, y, t-s) u_{2}(s, y) d s d y\right. \\
& \left.-b_{1} \int_{\Omega} \int_{-\infty}^{t} K_{1}(x, y, t-s) u_{2}(s, y) d s d y\right), \\
\frac{\partial u_{2}}{\partial t}= & d_{2} \Delta u_{2}+u_{2}\left(r_{2}-b_{2} \int_{\Omega} \int_{-\infty}^{t} K_{2}(x, y, t-s) u_{1}(s, y) d s d y\right. \\
& \left.-a_{2} u_{2}-c_{2} \int_{\Omega} \int_{-\infty}^{t} H_{2}(x, y, t-s) u_{1}(s, y) d s d y\right),
\end{aligned}
$$

where $a_{i} \geq 0, b_{i}>0, c_{i} \geq 0, i=1,2$, are constants and the kernels $H_{i}, i=1,2$, satisfy similar properties as the $K_{i}$ of (1.1). Notice that system (1.1) is a special case of (2.15) with $c_{i}=0$. When $a_{i}=0$, even when there is no diffusion (i.e., the ordinary delay competition model), both stability (see Gomatam and MacDonald [5]) 
and bifurcation (see Gopalsamy and Aggarwala [7]) are possible. We anticipate that system (2.15) will exhibit more complex dynamics, such as Hopf bifurcations, and we leave this for future consideration.

3. Travelling front solutions. In this section we discuss the modifications necessary to system (1.1) for the case of an infinite one-dimensional domain $\Omega=$ $(-\infty, \infty)$, and travelling front solutions of the resulting system. The infinite domain case is in some respects slightly simpler from a modelling point of view since there are no boundaries for individuals to interact with as they drift from their past to their present positions. Because of this, in contrast to the finite domain case, the nonlocal averaging associated with the delay takes the form of a spatial convolution, so that the model assumes the form

$$
\begin{aligned}
& \frac{\partial u_{1}}{\partial t}=d_{1} \frac{\partial^{2} u_{1}}{\partial x^{2}}+u_{1}\left(r_{1}-a_{1} u_{1}-b_{1} \int_{-\infty}^{\infty} \int_{-\infty}^{t} G_{1}(x-y, t-s) k_{1}(t-s) u_{2}(s, y) d s d y\right), \\
& \frac{\partial u_{2}}{\partial t}=d_{2} \frac{\partial^{2} u_{2}}{\partial x^{2}}+u_{2}\left(r_{2}-b_{2} \int_{-\infty}^{\infty} \int_{-\infty}^{t} G_{2}(x-y, t-s) k_{2}(t-s) u_{1}(s, y) d s d y-a_{2} u_{2}\right),
\end{aligned}
$$

where the $k_{i}$ satisfy $\int_{0}^{\infty} k_{i}(s) d s=1, i=1,2$, and the $G_{i}$ satisfy diffusion equations as in Lemma 2.1 but without the boundary conditions. To be more precise, $G_{1}$ is a weighting function describing the distribution at past times of the individuals of the species $u_{2}$ who are at position $x$ at time $t$. The $u_{2}$ individuals diffuse at diffusivity $d_{2}$; thus $G_{1}$ must satisfy

$$
\frac{\partial G_{1}}{\partial t}=d_{2} \frac{\partial^{2} G_{1}}{\partial x^{2}}, \quad G_{1}(x, 0)=\delta(x),
$$

and similarly, $G_{2}$ satisfies

$$
\frac{\partial G_{2}}{\partial t}=d_{1} \frac{\partial^{2} G_{2}}{\partial x^{2}}, \quad G_{2}(x, 0)=\delta(x),
$$

so that $G_{1}, G_{2}$ are both fundamental solutions of heat equations. With these assumptions, system (3.1) still preserves the same equilibria $E_{0}, E_{1}, E_{2}$, and (possibly) $E^{*}$ enumerated earlier.

Our interest in this section is in the possibility of a transition between the boundary equilibria $E_{1}$ and $E_{2}$ in the form of a travelling wave-front solution. This is of ecological interest since it corresponds to a situation where an environment is initially inhabited only by the weaker of the two competitors at its carrying capacity, and some of the stronger competitor are introduced and then invade the domain, dominate, and drive the weaker to extinction so that the end result is that only the stronger species is present, at its carrying capacity.

In this section the assumptions we shall make on the parameters are those which ensure that the corresponding system without diffusion and without delay (removal of delay can be effected by setting each $k_{i}(t)=\delta(t)$ in (3.1)) has $E_{1}$ unstable and $E_{2}$ asymptotically stable. Elementary analysis shows that the conditions for this to happen are

$$
r_{1} b_{2}<r_{2} a_{1} \quad \text { and } \quad r_{1} a_{2}<r_{2} b_{1} .
$$


Note that if (3.2) is satisfied, then the coexistence equilibrium $E^{*}$ is absent. In the two-dimensional $\left(u_{1}, u_{2}\right)$ phase plane, the diffusionless undelayed ODEs possess a heteroclinic connection from $E_{1}$ to $E_{2}$. It is known from the papers referred to in the introduction that under these circumstances the (undelayed) reaction-diffusion system has travelling-front solutions connecting these equilibria. Our intention now is to prove, for certain choices of the kernels $k_{i}$, that these travelling fronts persist under the introduction of delay, at least for small delays.

We shall consider the situation when the kernels $k_{i}$ are given by

$$
k_{1}(t)=\frac{1}{\tau_{1}} e^{-t / \tau_{1}}, \quad k_{2}(t)=\frac{1}{\tau_{2}} e^{-t / \tau_{2}},
$$

where the delays $\tau_{1}, \tau_{2}>0$, and we shall prove the following.

THEOREM 3.1. Let $k_{1}$ and $k_{2}$ be given by (3.3) and assume that (3.2) holds. Then, for sufficiently small delays $\tau_{1}, \tau_{2}$, system (3.1) possesses travelling front solutions connecting the semitrivial equilibria $E_{1}=\left(r_{1} / a_{1}, 0\right)$ and $E_{2}=\left(0, r_{2} / a_{2}\right)$.

Proof. With the kernels given by (3.3), it is straightforward to see that system (3.1) is equivalent to

$$
\begin{aligned}
& \frac{\partial u_{1}}{\partial t}=d_{1} \frac{\partial^{2} u_{1}}{\partial x^{2}}+u_{1}\left(r_{1}-a_{1} u_{1}-b_{1} w_{1}\right), \\
& \frac{\partial u_{2}}{\partial t}=d_{2} \frac{\partial^{2} u_{2}}{\partial x^{2}}+u_{2}\left(r_{2}-b_{2} w_{2}-a_{2} u_{2}\right), \\
& \frac{\partial w_{1}}{\partial t}=d_{2} \frac{\partial^{2} w_{1}}{\partial x^{2}}+\frac{1}{\tau_{1}} u_{2}-\frac{1}{\tau_{1}} w_{1}, \\
& \frac{\partial w_{2}}{\partial t}=d_{1} \frac{\partial^{2} w_{2}}{\partial x^{2}}+\frac{1}{\tau_{2}} u_{1}-\frac{1}{\tau_{2}} w_{2} .
\end{aligned}
$$

Converting to travelling wave form, by writing

$$
u_{1}(t, x)=u_{1}(z), \quad z=x+c t
$$

and similarly for the other state variables, gives

$$
\begin{aligned}
& c u_{1}^{\prime}=d_{1} u_{1}^{\prime \prime}+u_{1}\left(r_{1}-a_{1} u_{1}-b_{1} w_{1}\right), \\
& c u_{2}^{\prime}=d_{2} u_{2}^{\prime \prime}+u_{2}\left(r_{2}-b_{2} w_{2}-a_{2} u_{2}\right), \\
& c w_{1}^{\prime}=d_{2} w_{1}^{\prime \prime}+\frac{1}{\tau_{1}} u_{2}-\frac{1}{\tau_{1}} w_{1}, \\
& c w_{2}^{\prime}=d_{1} w_{2}^{\prime \prime}+\frac{1}{\tau_{2}} u_{1}-\frac{1}{\tau_{2}} w_{2},
\end{aligned}
$$

where prime denotes differentiation with respect to $z$. Let us introduce

$$
v_{1}=d_{1} u_{1}^{\prime}, \quad v_{2}=d_{2} u_{2}^{\prime}, \quad v_{3}=d_{2} w_{1}^{\prime}, \quad v_{4}=d_{1} w_{2}^{\prime} .
$$

Also, we shall replace $\tau_{1}$ and $\tau_{2}$ with $\varepsilon^{2} \tau_{1}$ and $\varepsilon^{2} \tau_{2}$, respectively, since we are interested 
in the situation when the delays are small. The system becomes

$$
\begin{aligned}
& u_{1}^{\prime}=\frac{1}{d_{1}} v_{1}, \\
& v_{1}^{\prime}=\frac{c}{d_{1}} v_{1}-u_{1}\left(r_{1}-a_{1} u_{1}-b_{1} w_{1}\right), \\
& u_{2}^{\prime}=\frac{1}{d_{2}} v_{2}, \\
& v_{2}^{\prime}=\frac{c}{d_{2}} v_{2}-u_{2}\left(r_{2}-b_{2} w_{2}-a_{2} u_{2}\right), \\
& w_{1}^{\prime}=\frac{1}{d_{2}} v_{3}, \\
& \varepsilon^{2} v_{3}^{\prime}=\frac{\varepsilon^{2} c}{d_{2}} v_{3}-\frac{1}{\tau_{1}} u_{2}+\frac{1}{\tau_{1}} w_{1}, \\
& w_{2}^{\prime}=\frac{1}{d_{1}} v_{4}, \\
& \varepsilon^{2} v_{4}^{\prime}=\frac{\varepsilon^{2} c}{d_{1}} v_{4}-\frac{1}{\tau_{2}} u_{1}+\frac{1}{\tau_{2}} w_{2} .
\end{aligned}
$$

If we introduce the new state variables

$\tilde{u}_{1}=u_{1}, \quad \tilde{v}_{1}=v_{1}, \quad \tilde{u}_{2}=u_{2}, \quad \tilde{v}_{2}=v_{2}, \quad \tilde{w}_{1}=w_{1}, \quad \tilde{v}_{3}=\varepsilon v_{3}, \quad \tilde{w}_{2}=w_{2}, \quad \tilde{v}_{4}=\varepsilon v_{4}$

and then drop the tildes, we have

$$
\begin{aligned}
& u_{1}^{\prime}=\frac{1}{d_{1}} v_{1}, \\
& v_{1}^{\prime}=\frac{c}{d_{1}} v_{1}-u_{1}\left(r_{1}-a_{1} u_{1}-b_{1} w_{1}\right), \\
& u_{2}^{\prime}=\frac{1}{d_{2}} v_{2}, \\
& v_{2}^{\prime}=\frac{c}{d_{2}} v_{2}-u_{2}\left(r_{2}-b_{2} w_{2}-a_{2} u_{2}\right), \\
& \varepsilon w_{1}^{\prime}=\frac{1}{d_{2}} v_{3}, \\
& \varepsilon v_{3}^{\prime}=\frac{\varepsilon c}{d_{2}} v_{3}-\frac{1}{\tau_{1}} u_{2}+\frac{1}{\tau_{1}} w_{1}, \\
& \varepsilon w_{2}^{\prime}=\frac{1}{d_{1}} v_{4}, \\
& \varepsilon v_{4}^{\prime}=\frac{\varepsilon c}{d_{1}} v_{4}-\frac{1}{\tau_{2}} u_{1}+\frac{1}{\tau_{2}} w_{2} .
\end{aligned}
$$

When $\varepsilon=0$, system (3.6) reduces to the equations satisfied by travelling wave solutions of the undelayed problem studied by previous investigators $[1,4,10,14]$. In this degenerate case the system is four-dimensional, but for $\varepsilon>0$ (i.e., delay is present), existence of a travelling front solution of (3.1) between $E_{1}$ and $E_{2}$ is equivalent to existence of a heteroclinic connection between the equilibrium points of the eightdimensional system (3.6) that correspond to $E_{1}$ and $E_{2}$ of (3.1). We shall still denote 
these equilibria by $E_{1}$ and $E_{2}$; for system (3.6) they are given by

$$
E_{1}=\left(\frac{r_{1}}{a_{1}}, 0,0,0,0,0, \frac{r_{1}}{a_{1}}, 0\right), \quad E_{2}=\left(0,0, \frac{r_{2}}{a_{2}}, 0, \frac{r_{2}}{a_{2}}, 0,0,0\right) .
$$

Our intention is to apply the geometric singular perturbation theory described in [3], in particular, Theorem 9.1 of that paper. System (3.6) above will henceforth be referred to as the slow system. By introducing a new independent variable $\eta$ defined by

$$
z=\varepsilon \eta
$$

system (3.6) transforms into

$$
\begin{aligned}
& \dot{u}_{1}=\frac{\varepsilon}{d_{1}} v_{1}, \\
& \dot{v}_{1}=\varepsilon\left(\frac{c}{d_{1}} v_{1}-u_{1}\left(r_{1}-a_{1} u_{1}-b_{1} w_{1}\right)\right), \\
& \dot{u}_{2}=\frac{\varepsilon}{d_{2}} v_{2}, \\
& \dot{v}_{2}=\varepsilon\left(\frac{c}{d_{2}} v_{2}-u_{2}\left(r_{2}-b_{2} w_{2}-a_{2} u_{2}\right)\right), \\
& \dot{w}_{1}=\frac{1}{d_{2}} v_{3}, \\
& \dot{v}_{3}=\frac{\varepsilon c}{d_{2}} v_{3}-\frac{1}{\tau_{1}} u_{2}+\frac{1}{\tau_{1}} w_{1}, \\
& \dot{w}_{2}=\frac{1}{d_{1}} v_{4}, \\
& \dot{v}_{4}=\frac{\varepsilon c}{d_{1}} v_{4}-\frac{1}{\tau_{2}} u_{1}+\frac{1}{\tau_{2}} w_{2},
\end{aligned}
$$

where dots denote differentiation with respect to $\eta$. System (3.8) is called the fast system. Geometric singular perturbation theory uses both the slow and the fast systems. The two are equivalent when $\varepsilon>0$, but when $\varepsilon=0$, the slow system (3.6) does not define a dynamical system in the whole of $\mathbf{R}^{8}$ but rather the dynamics takes place only on

$$
M_{0}=\left\{\left(u_{1}, v_{1}, u_{2}, v_{2}, w_{1}, v_{3}, w_{2}, v_{4}\right) \in \mathbf{R}^{8}: \quad v_{3}=0, \quad v_{4}=0, \quad w_{1}=u_{2}, \quad w_{2}=u_{1}\right\},
$$

which is a four-dimensional submanifold of $\mathbf{R}^{8}$. Note that $M_{0}$ consists of the equilibria of the fast system when $\varepsilon=0$. If $M_{0}$ is normally hyperbolic then, for sufficiently small $\varepsilon>0$, Theorem 9.1 in [3] provides us with a four-dimensional invariant manifold $M_{\varepsilon}$ for the system (3.6). It will be shown that the equilibrium points $E_{1}$ and $E_{2}$ lie on $M_{\varepsilon}$. By studying the system (3.6) reduced to this manifold, the dimensionality is reduced back to four and the existence of the heteroclinic connection we are seeking can be established.

To verify normal hyperbolicity it is necessary to use the fast system (3.8). We need to verify that the linearization of (3.8), restricted to $M_{0}$, has exactly four (= $\operatorname{dim} M_{0}$ ) 
eigenvalues on the imaginary axis with the remainder of the spectrum hyperbolic. The linearization of the fast system, when $\varepsilon=0$, is given by

$$
\left(\begin{array}{cccccccc}
0 & 0 & 0 & 0 & 0 & 0 & 0 & 0 \\
0 & 0 & 0 & 0 & 0 & 0 & 0 & 0 \\
0 & 0 & 0 & 0 & 0 & 0 & 0 & 0 \\
0 & 0 & 0 & 0 & 0 & 0 & 0 & 0 \\
0 & 0 & 0 & 0 & 0 & \frac{1}{d_{2}} & 0 & 0 \\
0 & 0 & -\frac{1}{\tau_{1}} & 0 & \frac{1}{\tau_{1}} & 0 & 0 & 0 \\
0 & 0 & 0 & 0 & 0 & 0 & 0 & \frac{1}{d_{1}} \\
-\frac{1}{\tau_{2}} & 0 & 0 & 0 & 0 & 0 & \frac{1}{\tau_{2}} & 0
\end{array}\right),
$$

which has eigenvalues $\left\{0,0,0,0, \pm 1 / \sqrt{\tau_{2} d_{1}}, \pm 1 / \sqrt{\tau_{1} d_{2}}\right\}$. Thus, normal hyperbolicity is verified and there exists an invariant manifold $M_{\varepsilon}$, close to $M_{0}$, for the perturbed system (3.6) for $\varepsilon>0$ sufficiently small. In fact, $M_{\varepsilon}$ can be expressed in the form

$$
\begin{gathered}
M_{\varepsilon}=\left\{\left(u_{1}, v_{1}, u_{2}, v_{2}, w_{1}, v_{3}, w_{2}, v_{4}\right) \in \mathbf{R}^{8}: \quad v_{3}=h_{1}\left(u_{1}, v_{1}, u_{2}, v_{2} ; \varepsilon\right),\right. \\
v_{4}=h_{2}\left(u_{1}, v_{1}, u_{2}, v_{2} ; \varepsilon\right), \quad w_{1}=u_{2}+h_{3}\left(u_{1}, v_{1}, u_{2}, v_{2} ; \varepsilon\right) \\
\left.w_{2}=u_{1}+h_{4}\left(u_{1}, v_{1}, u_{2}, v_{2} ; \varepsilon\right)\right\}
\end{gathered}
$$

with $h_{i}\left(u_{1}, v_{1}, u_{2}, v_{2} ; 0\right)=0$ for $i=1,2,3,4$. The $h_{i}$ can be computed by substitution into (3.6).

The slow system (3.6), restricted to $M_{\varepsilon}$, is

$$
\begin{aligned}
& u_{1}^{\prime}=\frac{1}{d_{1}} v_{1}, \\
& v_{1}^{\prime}=\frac{c}{d_{1}} v_{1}-u_{1}\left(r_{1}-a_{1} u_{1}-b_{1} u_{2}-b_{1} h_{3}\left(u_{1}, v_{1}, u_{2}, v_{2} ; \varepsilon\right)\right), \\
& u_{2}^{\prime}=\frac{1}{d_{2}} v_{2}, \\
& v_{2}^{\prime}=\frac{c}{d_{2}} v_{2}-u_{2}\left(r_{2}-a_{2} u_{2}-b_{2} u_{1}-b_{2} h_{4}\left(u_{1}, v_{1}, u_{2}, v_{2} ; \varepsilon\right)\right) .
\end{aligned}
$$

When $\varepsilon=0$ (i.e., no delay), system (3.11) again reduces to the system satisfied by travelling wave solutions of the undelayed equations, which has been studied previously. What we now claim is that, for $\varepsilon>0$ sufficiently small, system (3.11) still possesses as equilibrium points

$$
E_{1}=\left(\frac{r_{1}}{a_{1}}, 0,0,0\right), \quad E_{2}=\left(0,0, \frac{r_{2}}{a_{2}}, 0\right)
$$

and that it falls within the class of systems studied by Gardner [4]. Neither is immediately clear. Indeed, Gardner studied competition systems of the form

$$
\begin{aligned}
& \partial u_{1} / \partial t=d_{1} \partial^{2} u_{1} / \partial x^{2}+u_{1} M\left(u_{1}, u_{2}\right), \\
& \partial u_{2} / \partial t=d_{2} \partial^{2} u_{2} / \partial x^{2}+u_{2} N\left(u_{1}, u_{2}\right),
\end{aligned}
$$


which, in travelling wave form, read as

$$
\begin{aligned}
u_{1}^{\prime} & =\frac{1}{d_{1}} v_{1}, \\
v_{1}^{\prime} & =\frac{c}{d_{1}} v_{1}-u_{1} M\left(u_{1}, u_{2}\right), \\
u_{2}^{\prime} & =\frac{1}{d_{2}} v_{2}, \\
v_{2}^{\prime} & =\frac{c}{d_{2}} v_{2}-u_{2} N\left(u_{1}, u_{2}\right) .
\end{aligned}
$$

Comparing (3.11) with (3.13) we see that, for Gardner's results to be applicable, the functions $h_{3}$ and $h_{4}$ in (3.11) would need to involve $u_{1}$ and $u_{2}$ only. We shall now show that this is indeed the case, up to order $\varepsilon^{2}$.

Indeed, straightforward but tedious calculations, utilizing the fact that $M_{\varepsilon}$ is an invariant manifold for (3.6), yield that the $h_{i}$ satisfy

$$
\begin{aligned}
& \varepsilon\left[\frac{1}{d_{2}} v_{2}+\frac{1}{d_{1}} v_{1} \frac{\partial h_{3}}{\partial u_{1}}+\frac{\partial h_{3}}{\partial v_{1}}\left(\frac{c}{d_{1}} v_{1}-u_{1}\left(r_{1}-a_{1} u_{1}-b_{1} u_{2}-b_{1} h_{3}\right)\right)\right. \\
& \left.+\frac{1}{d_{2}} v_{2} \frac{\partial h_{3}}{\partial u_{2}}+\frac{\partial h_{3}}{\partial v_{2}}\left(\frac{c}{d_{2}} v_{2}-u_{2}\left(r_{2}-a_{2} u_{2}-b_{2} u_{1}-b_{2} h_{4}\right)\right)\right]=\frac{1}{d_{2}} h_{1}
\end{aligned}
$$

together with three other similar equations. Attempting solutions of the equations in the form

$$
h_{1}\left(u_{1}, v_{1}, u_{2}, v_{2} ; \varepsilon\right)=\varepsilon h_{1}^{(1)}\left(u_{1}, v_{1}, u_{2}, v_{2}\right)+\varepsilon^{2} h_{1}^{(2)}\left(u_{1}, v_{1}, u_{2}, v_{2}\right)+\cdots,
$$

and similarly for the other $h_{i}$, yields, after some further algebra, that

$$
h_{1}^{(1)}=v_{2}, \quad h_{2}^{(1)}=v_{1}, \quad h_{3}^{(1)}=0, \quad h_{4}^{(1)}=0
$$

and

$$
\begin{gathered}
h_{1}^{(2)}=0, \quad h_{2}^{(2)}=0, \\
h_{3}^{(2)}=-\tau_{1} u_{2}\left(r_{2}-a_{2} u_{2}-b_{2} u_{1}\right), \\
h_{4}^{(2)}=-\tau_{2} u_{1}\left(r_{1}-a_{1} u_{1}-b_{1} u_{2}\right) .
\end{gathered}
$$

Thus, system (3.11) becomes, to order $\varepsilon^{2}$,

$$
\begin{aligned}
& u_{1}^{\prime}=\frac{1}{d_{1}} v_{1} \\
& v_{1}^{\prime}=\frac{c}{d_{1}} v_{1}-u_{1}\left(r_{1}-a_{1} u_{1}-b_{1} u_{2}+\varepsilon^{2} b_{1} \tau_{1} u_{2}\left(r_{2}-a_{2} u_{2}-b_{2} u_{1}\right)\right), \\
& u_{2}^{\prime}=\frac{1}{d_{2}} v_{2} \\
& v_{2}^{\prime}=\frac{c}{d_{2}} v_{2}-u_{2}\left(r_{2}-a_{2} u_{2}-b_{2} u_{1}+\varepsilon^{2} b_{2} \tau_{2} u_{1}\left(r_{1}-a_{1} u_{1}-b_{1} u_{2}\right)\right),
\end{aligned}
$$

which has the structure of the system (3.13). Also, note that $E_{1}$ and $E_{2}$, given by (3.12), are indeed equilibria of (3.14). Therefore, the results in [4] are applicable, 
yielding a heteroclinic connection between the equilibria $E_{1}$ and $E_{2}$ of (3.14). We have shown that travelling fronts exist for system (3.1) when the kernels $k_{1}$ and $k_{2}$ are given by (3.3), so the proof of Theorem 3.1 is complete.

Remark 3.2. Let us briefly discuss the role of the terms of order $\varepsilon^{2}$. If the system (3.14) is linearized about the equilibrium $E_{2}$, we find that the eigenvalues $\lambda$ of the linearization satisfy an equation that does not involve $\varepsilon$, namely

$$
\left(d_{2} \lambda^{2}-c \lambda-r_{2}\right)\left(d_{1} a_{2} \lambda^{2}-c a_{2} \lambda+r_{1} a_{2}-r_{2} b_{1}\right)=0 .
$$

About the equilibrium $E_{1}$, the eigenvalue equation becomes

$$
\left(d_{1} \lambda^{2}-c \lambda-r_{1}\right)\left(d_{2} a_{1} \lambda^{2}-c a_{1} \lambda+r_{2} a_{1}-r_{1} b_{2}\right)=0,
$$

which again does not involve $\varepsilon$. These observations suggest that the manner in which the front approaches the equilibria $E_{1}$ and $E_{2}$ as $z \rightarrow-\infty$ and $z \rightarrow \infty$, respectively, is independent of $\varepsilon$ for $\varepsilon>0$ sufficiently small and therefore that the front's qualitative profile is not sensitive to the delays, provided they are both sufficiently small. Of course, system (3.14) is itself the result of a perturbation analysis for small $\varepsilon$, and therefore no conclusions can be drawn for larger $\varepsilon$. In conclusion, we may state that the travelling front solutions of the corresponding undelayed competition model appear to be very robust, not only in the sense that they persist under the introduction of delays, but also in that they are not sensitive to small delays in the sense that, if the delays are small, they look qualitatively the same as they do with no delay at all.

\section{REFERENCES}

[1] C. Conley and R. Gardner, An application of the generalized Morse index to travelling wave solutions of a competitive reaction-diffusion model, Indiana Univ. Math. J., 33 (1984), pp. 319-343.

[2] J. M. Cushing, Integrodifferential Equations and Delay Models in Population Dynamics, Springer-Verlag, Heidelberg, 1977.

[3] N. FENICHEL, Geometric singular perturbation theory for ordinary differential equations, J. Differential Equations, 31 (1979), pp. 53-98.

[4] R. GARDNER, Existence and stability of travelling wave solutions of competition models: A degree theoretic approach, J. Differential Equations, 44 (1982), pp. 343-364.

[5] J. Gomatam and N. MacDonald, Time delays and stability of two competing species, Math. Biosci., 24 (1975), pp. 247-255.

[6] K. Gopalsamy, Time lags and global stability in two-species competition, Bull. Math. Biol., 42 (1980), pp. 729-737.

[7] K. Gopalsamy and B. D. Aggarwala, Recurrence and non-stationary coexistence in twospecies competition, Ecol. Model., 9 (1980), pp. 153-163.

[8] S. A. Gourley and N. F. Britton, A predator prey reaction diffusion system with nonlocal effects, J. Math. Biol., 34 (1996), pp. 297-333.

[9] S. A. Gourley AND J. W.-H. So, Dynamics of a food-limited population model incorporating nonlocal delays on a finite domain, J. Math. Biol., 44 (2002), pp. 49-78.

[10] Y. KAN-ON, Parameter dependence of propagation speed of travelling waves for competitiondiffusion equations, SIAM J. Math. Anal., 26 (1995), pp. 340-363.

[11] S. Ruan And J. Wu, Reaction-diffusion systems with infinite delay, Canad. Appl. Math. Quart., 2 (1994), pp. 485-550.

[12] S. Ruan And X.-Q. ZhaO, Persistence and extinction in two species reaction-diffusion systems with delays, J. Differential Equations, 156 (1999), pp. 71-92.

[13] A. Schiaffino And A. Tesei, Competition systems with Dirichlet boundary conditions, J. Math. Biol., 15 (1982), pp. 93-105.

[14] M. M. TANG AND P. C. Fife, Propagating fronts for competing species equations with diffusion, Arch. Ration. Mech. Anal., 73 (1980), pp. 69-77.

[15] J. Wu, Theory and Applications of Partial Functional Differential Equations, Springer-Verlag, New York, 1996. 
[16] Y. YAmadA, On a certain class of semilinear Volterra diffusion equations, J. Math. Anal. Appl., 88 (1982), pp. 433-451.

[17] Y. Yamada, Asymptotic stability for some systems of semilinear Volterra diffusion equations, J. Differential Equations, 52 (1984), pp. 295-326.

[18] L. ZhOU AND C. V. PAO, Asymptotic behavior of a competition-diffusion system in population dynamics, Nonlinear Anal., 6 (1982), pp. 1163-1184. 